\title{
ON PROJECTION BODIES OF ORDER ONE
}

\author{
STEFANO CAMPI AND PAOLO GRONCHI
}

\begin{abstract}
The projection body of order one $\Pi_{1} K$ of a convex body $K$ in $\mathbb{R}^{n}$ is the body whose support function is, up to a constant, the average mean width of the orthogonal projections of $K$ onto hyperplanes through the origin.

The paper contains an inequality for the support function of $\Pi_{1} K$, which implies in particular that such a function is strictly convex, unless $K$ is one or two dimensional. Furthermore, an existence problem related to the reconstruction of a convex body is discussed to highlight the different behavior of the area measures of order one and of order $n-1$.
\end{abstract}

\section{INTRODUCTION}

This paper investigates some properties of projection bodies of order one. Projection bodies are special sets which play an important role in convex geometry. We refer to Schneider's treatise [11] for information about this area.

By a convex body we mean here a compact convex subset of $\mathbb{R}^{n}$. The support function of a convex body $K$ is defined by

$$
h_{K}(x)=\max _{y \in K}\langle x, y\rangle, \text { for } x \in \mathbb{R}^{n},
$$

where $\langle\cdot, \cdot\rangle$ denotes the standard scalar product in $\mathbb{R}^{n}$. Note that $h_{K}$ is positively homogeneous and so it is determined everywhere by its values on the unit sphere $S^{n-1}$ of $\mathbb{R}^{n}$.

By definition, the Minkowski sum of two convex bodies $K$ and $L$ is the convex body $K+L$ such that

$$
h_{K+L}(x)=h_{K}(x)+h_{L}(x) .
$$

In particular, the difference body $D K$ of $K$ is the Minkowski sum of $K$ and its reflection $-K$ in the origin. Clearly $D K$ is origin symmetric.

The intrinsic volumes are quantities naturally associated to a given convex body which can be introduced in different ways. For instance (see [11], p. 210 and p. 295), the $i$ th intrinsic volume of $K$ in $\mathbb{R}^{n}, 0 \leq i \leq n$, is defined by

$$
V_{i}(K)=\left(\begin{array}{c}
n \\
i
\end{array}\right) \frac{\kappa_{n}}{\kappa_{i} \kappa_{n-i}} \int_{G(n, i)} \lambda_{i}(K \mid S) d S,
$$

where $\kappa_{i}$ denotes the volume of the unit ball in $\mathbb{R}^{i}, \lambda_{i}$ the $i$-dimensional Lebesgue measure in $\mathbb{R}^{i}, G(n, i)$ the Grassmann manifold of $i$-dimensional subspaces of $\mathbb{R}^{n}, K \mid S$ the orthogonal projection of $K$ onto $S$ and integration is with respect to the Haar probability measure.

2000 Mathematics Subject Classification. 52A40. 
Special cases are $V_{n}$, which is the standard volume, $V_{n-1}$, one half of the surface area, and $V_{1}$, the mean width, up to the constant $n \kappa_{n} / 2 \kappa_{n-1}$.

For every convex body $K$ the $i$ th intrinsic volume of the projection of $K$ in the direction $u$ turns out to be the support function of a convex body. The projection body $\Pi_{i} K$ of order $i$ of $K$ is just the body defined by

$$
h_{\Pi_{i} K}(x)=\frac{\|x\| \kappa_{n-1-i}}{\left(\begin{array}{c}
n-1 \\
i
\end{array}\right)} V_{i}\left(K \mid x^{\perp}\right),
$$

where $\|\cdot\|$ denotes the usual norm in $\mathbb{R}^{n}$ and $x^{\perp}$ the hyperplane through the origin orthogonal to $x$. For fixed $i$, to every convex body $K$ is associated a Borel measure $S_{i}(K ; \cdot)$ on $S^{n-1}$, the $i$ th order area measure of $K$, such that, for $x \in S^{n-1}$,

$$
V_{i}\left(K \mid x^{\perp}\right)=\frac{\left(\begin{array}{c}
n-1 \\
i
\end{array}\right)}{2 \kappa_{n-1-i}} \int_{S^{n-1}}|\langle x, z\rangle| d S_{i}(K ; z)
$$

(see [11], p. 210 and p. 421). In the case of a smooth convex body $K$, the measure $S_{i}(K ; \cdot)$ is absolutely continuous with respect to Hausdorff measure and its density is the $i$ th normalized elementary symmetric function of the radii of curvature of $\partial K$ (see [11], Sect. 5.3).

Combining (1) and (2) yields

$$
h_{\Pi_{i} K}(x)=\frac{1}{2} \int_{S^{n-1}}|\langle x, z\rangle| d S_{i}(K ; z),
$$

which says that $h_{\Pi_{i} K}$ is the cosine transform of the measure $S_{i}(K ; \cdot)$ (see, for instance, [8], p. 97 and [11], p. 421).

The class of projection bodies of order $n-1$ has been widely investigated and it is closely related to the class of zonoids, which are limits, in the Hausdorff metric, of sequences of zonotopes, i.e. of finite Minkowski sums of segments (for a survey on this topic we refer to [12]). Precisely, every projection body of order $n-1$ is a zonoid. The converse is true when the zonoid is full dimensional.

Every projection body of order $i$ is a zonoid, but nontrivial characterizations are not available when $i<n-1$.

The interest in projection bodies was renewed by recent applications in Geometric Tomography. For exhaustive and updated information on this subject, see Gardner's book [6].

In this paper (Theorem 2.1) we provide a necessary condition for a function to be the support function of a projection body of order one.

By definition of $V_{1}$ and (1), the support function of $\Pi_{1} K$ can be expressed as

$$
h_{\Pi_{1} K}(x)=\frac{\|x\|}{(n-1)} \int_{S^{n-1} \cap x^{\perp}} h_{K}(z) d z,
$$


which implies the following linearity property:

$$
\Pi_{1}(K+L)=\Pi_{1} K+\Pi_{1} L .
$$

By (4), $h_{\Pi_{1} K}$ can be seen as the spherical Radon transform of $h_{K}$ (see, for instance, [8] p. 98). Since $\Pi_{1} K=\Pi_{1}(-K)$, equality (5) implies that $2 \Pi_{1} K=\Pi_{1}(D K)$. Therefore, every projection body of order one comes from an origin-symmetric convex body. In what follows we shall restrict the discussion to the class of origin-symmetric bodies.

For $n=2, \Pi_{1} K$ is nothing but a rotated copy of the difference body $D K$ and we shall not deal with this case.

For $n=3$, the integral in (4) is just the perimeter of the projection of $K$ onto $x^{\perp}$.

When $K$ is a segment of $\mathbb{R}^{n}$ with length $\ell$, the corresponding $\Pi_{1} K$ is an $(n-1)$-dimensional ball centered at the origin, with radius $\ell \kappa_{n-2} /(n-1)$, lying on the hyperplane orthogonal to $K$. Indeed, $\Pi_{1} K$ inherits all symmetries of $K$ and one projection of $K$ reduces to a point, whose first intrinsic volume trivially vanishes.

When $K$ is a zonotope, by the linearity property (5), $\Pi_{1} K$ is a sum of $(n-1)$-dimensional balls.

This case provides a lot of examples of projection bodies of order one and suggests that in general $\partial \Pi_{1} K$ has no singular points, and hence that the support function of $\Pi_{1} K$ is more regular than that of an arbitrary zonoid.

This is just what we prove in Section 2, where we show that the support function of $\Pi_{1} K$ is strictly convex (unless $K$ is one or two dimensional). This result is achieved by an inequality which in turn implies an estimate from below of quantities related to the radii of curvature of $\partial \Pi_{1} K$ in terms of some particular values of $h_{K}$.

Section 3 is devoted to comparing the class of projection bodies of first order with that of order $n-1$, through the study of the existence of special convex bodies whose projection bodies satisfy given conditions. To this end, let us consider the following general problem:

Problem 1.1. Find an origin-symmetric convex body $K$ (or possibly an approximation of $K$ ), given the $i$ th intrinsic volumes of the orthogonal projections of $K$ onto all (or possibly some, respectively) hyperplanes.

First, let us assume that the data are available for all projections. Then, by (3), the even part of the $i$ th area measure can be recovered (see [8], Sect. 3.4).

The problem of existence and uniqueness of a convex body having a given $i$ th area measure is called the Minkowski problem of order $i$, for $i \neq 1$, and the Christoffel problem, for $i=1$. It is well known (see for instance [11], Corollary 7.2.5) that for every $i, 1 \leq i \leq n-1$, the solution is unique. As far as the existence is concerned, a necessary condition for a measure $\mu$ to be the $i$ th area measure of a convex body is

$$
\int_{S^{n-1}} z d \mu(z)=0 \text {. }
$$

Such a condition is also sufficient only for $i=n-1$. In the case $i=1$, necessary and sufficient conditions were found by Firey [5] and Berg [1] (see also [11], Th. 4.3.4). Recently, Guan and Ma [9] found sufficient conditions easy to handle for the area measure of every order. 
Let us assume now that the data are given only for finitely many hyperplanes, orthogonal to the directions $u_{1}, u_{2}, \ldots, u_{s}$, and consider the case of the brightness, i.e. of the $(n-1)$-volume of the projections. In [3] it is shown that among all convex bodies with the same brightness as $K$ along $u_{1}, u_{2}, \ldots, u_{s}$, the element of maximal volume is a polytope. Furthermore, its area measure is concentrated on the nodes of the given directions, where by node we mean here a direction orthogonal to $n-1$ of the $u_{j}$ 's.

The existence of such a polytope was used by Gardner and Milanfar [7] to develop an algorithm for reconstructing arbitrarily close approximations to an origin-symmetric convex body from finitely many (even noisy) values of its brightness function, thus solving completely Problem 1.1 when $i=n-1$.

We prove here that even in $\mathbb{R}^{3}$ the existence of a polytope $P$ whose area measure is concentrated on the nodes of the $u_{j}$ 's and such that $V_{1}\left(P \mid u_{j}^{\perp}\right)=V_{1}\left(K \mid u_{j}^{\perp}\right)$, for all $j$, is not guaranteed for every $K$. This is done by a suitable choice of the set of directions, which leads to rephrasing the existence problem in terms of an inequality for the support function of $\Pi_{1} K$. It is worth noting that such an inequality is of the same kind as that proved in Theorem 2.1, but it does not hold for every $K$.

\section{StRict CONVEXity of $h_{\Pi_{1} K}$}

This section is devoted to showing that for every convex body $K$, the support function of the projection body of the first order of $K$ is strictly convex, unless $K$ is one or two dimensional.

Theorem 2.1. Let $K$ be a convex body in $\mathbb{R}^{n}$, and let $u_{1}, u_{2} \in S^{n-1}, u_{1} \neq u_{2}$. Then

$$
h_{\Pi_{1} K}\left(u_{1}\right)+h_{\Pi_{1} K}\left(u_{2}\right)-h_{\Pi_{1} K}\left(u_{1}+u_{2}\right) \geq \frac{2\left(2-\left\|u_{1}+u_{2}\right\|\right)(n-3) ! !}{(n-1)(n-2) ! !} I\left(K ; u_{1}, u_{2}\right)
$$

where

$$
I\left(K ; u_{1}, u_{2}\right)=\int_{S^{n-1} \cap u_{1}^{\perp} \cap u_{2}^{\perp}} \frac{h_{K}(z)^{2}}{h_{K}(v)+\sqrt{h_{K}(v)^{2}+h_{K}(z)^{2}}} d z \quad, \quad v=\frac{u_{1}-u_{2}}{\left\|u_{1}-u_{2}\right\|} .
$$

Proof. The proof is based on formula (4). By taking into account the homogeneity of $h_{K}$, the integration is performed on a suitable linear subspace and the result follows using the convexity of $h_{K}$.

Choose a Cartesian coordinate system so that $u_{1}=(\cos \theta, \sin \theta, 0, \ldots, 0)$ and $u_{2}=(\cos \theta,-\sin \theta, 0, \ldots, 0)$ $\theta \in(0, \pi / 2)$. We introduce suitable polar coordinates $\varphi_{1} \in[0,2 \pi), \varphi_{2}, \varphi_{3}, \ldots, \varphi_{n-1} \in$ 
$[-\pi / 2, \pi / 2]$, so that

$$
\begin{cases}x_{1}= & \cos \varphi_{n-1} \cos \varphi_{n-2} \cdots \cos \varphi_{2} \cos \varphi_{1} \\ x_{2}= & \cos \varphi_{n-1} \cos \varphi_{n-2} \cdots \cos \varphi_{2} \sin \varphi_{1} \\ x_{3}= & \cos \varphi_{n-1} \cos \varphi_{n-2} \cdots \cos \varphi_{3} \sin \varphi_{2} \\ \vdots & \vdots \\ x_{k}= & \cos \varphi_{n-1} \cdots \cos \varphi_{k} \sin \varphi_{k-1} \\ \vdots & \vdots \\ x_{n}= & \sin \varphi_{n-1}\end{cases}
$$

and new variables $y_{2}, y_{3}, \ldots, y_{n-1}$ defined by

$$
\left\{\begin{array}{ll}
y_{2}= & \frac{\tan \varphi_{2}}{\cos \theta} \\
y_{3}= & \frac{\tan \varphi_{3}}{\cos \theta \cos \varphi_{2}} \\
y_{4}= & \frac{\tan \varphi_{4}}{\cos \theta \cos \varphi_{2} \cos \varphi_{3}} \\
\vdots & \vdots \\
y_{n-1}= & \frac{\tan \varphi_{n-1}}{\cos \theta \cos \varphi_{2} \cdots \cos \varphi_{n-2}}
\end{array} .\right.
$$

If we denote by $\left|\frac{\partial x_{i}}{\partial \varphi_{j}}\right|$ and $\left|\frac{\partial \varphi_{i}}{\partial y_{j}}\right|$ the determinant, in absolute value, of the Jacobian matrix of the above changes of variables, respectively, then we can write

$$
\begin{gathered}
h_{\Pi_{1} K}\left(u_{1}\right)=\frac{2}{n-1} \int_{\left[-\frac{\pi}{2}, \frac{\pi}{2}\right]^{n-2}} h_{K}\left(x\left(\theta+\frac{\pi}{2}, \varphi_{2}, \ldots, \varphi_{n-1}\right)\right)\left|\frac{\partial x_{i}}{\partial \varphi_{j}}\right| d \varphi_{2} \cdots d \varphi_{n-1} \\
=\frac{2}{n-1} \int_{\mathbb{R}^{n-2}} h_{K}\left(-\tan \theta, 1, y_{2}, y_{3}, \ldots, y_{n-1}\right) \cos \theta \cos \varphi_{2} \cdots \cos \varphi_{n-1}\left|\frac{\partial x_{i}}{\partial \varphi_{j}}\right|\left|\frac{\partial \varphi_{i}}{\partial y_{j}}\right| d y_{2} \cdots d y_{n-1} \\
=\frac{2}{n-1} \int_{\mathbb{R}^{n-2}} h_{K}\left(-\tan \theta, 1, y_{2}, y_{3}, \ldots, y_{n-1}\right) \frac{\cos ^{n-1} \theta}{\left[1+\cos ^{2} \theta \sum_{k=2}^{n-1} y_{k}^{2}\right]^{\frac{n}{2}}} d y_{2} \cdots d y_{n-1},
\end{gathered}
$$

where $x=\left(x_{1}, x_{2}, \ldots, x_{n}\right)$ and we make use of (7), (8), as well as the homogeneity of $h_{K}$ and the assumption $h_{K}(z)=h_{K}(-z)$. 
An analogous expression holds for $h_{\Pi_{1} K}\left(u_{2}\right)$. For $h_{\Pi_{1} K}\left(u_{1}+u_{2}\right)$, by (4) again, we get

$$
\begin{gathered}
h_{\Pi_{1} K}\left(u_{1}+u_{2}\right)=\frac{2\left\|u_{1}+u_{2}\right\|}{n-1} \int_{\left[-\frac{\pi}{2}, \frac{\pi}{2}\right]^{n-2}} h_{K}\left(x\left(\frac{\pi}{2}, \varphi_{2}, \ldots, \varphi_{n-1}\right)\right)\left|\frac{\partial x_{i}}{\partial \varphi_{i}}\right| d \varphi_{2} \cdots d \varphi_{n-1} \\
=\frac{4 \cos \theta}{n-1} \int_{\mathbb{R}^{n-2}} h_{K}\left(0, \frac{1}{\cos \theta}, y_{2}, y_{3}, \ldots, y_{n-1}\right) \cos \theta \cos \varphi_{2} \cdots \cos \varphi_{n-1}\left|\frac{\partial x_{i}}{\partial \varphi_{i}}\right|\left|\frac{\partial \varphi_{i}}{\partial y_{i}}\right| d y_{2} \cdots d y_{n-1} \\
=\frac{4}{n-1} \int_{\mathbb{R}^{n-2}} h_{K}\left(0,1, \bar{y}_{2}, \bar{y}_{3}, \ldots, \bar{y}_{n-1}\right) \frac{\cos \theta}{\left[1+\sum_{k=2}^{n-1} \bar{y}_{k}^{2}\right]^{\frac{n}{2}}} d \bar{y}_{2} \cdots d \bar{y}_{n-1},
\end{gathered}
$$

where we performed the substitutions $\bar{y}_{i}=y_{i} \cos \theta$, for $i=2,3, \ldots, n-1$.

The sublinearity of $h_{K}$ implies that

$$
h_{K}\left(-\tan \theta, 1, y_{2}, y_{3}, \ldots, y_{n-1}\right)+h_{K}\left(\tan \theta, 1, y_{2}, y_{3}, \ldots, y_{n-1}\right) \geq 2 h_{K}\left(0,1, y_{2}, y_{3}, \ldots, y_{n-1}\right)
$$

and so we obtain the inequality

$$
\begin{aligned}
& h_{\Pi_{1} K}\left(u_{1}\right)+h_{\Pi_{1} K}\left(u_{2}\right)-h_{\Pi_{1} K}\left(u_{1}+u_{2}\right) \geq \\
& \quad \frac{4}{n-1} \int_{\mathbb{R}^{n-2}} h_{K}\left(0,1, y_{2}, y_{3}, \ldots, y_{n-1}\right)\left(\frac{\cos ^{n-1} \theta}{\left[1+\cos ^{2} \theta \sum_{k=2}^{n-1} y_{k}^{2}\right]^{\frac{n}{2}}}-\frac{\cos \theta}{\left[1+\sum_{k=2}^{n-1} y_{k}^{2}\right]^{\frac{n}{2}}}\right) d y_{2} \cdots d y_{n-1} .
\end{aligned}
$$

Now, we introduce polar coordinates $r \in \mathbb{R}$ and $z \in S^{n-3}$ in the $(n-2)$-dimensional subspace where the integration is made and denote $h_{K}\left(0,1, y_{2}(r, z), \ldots, y_{n-1}(r, z)\right)$ by $H_{K}(r, z)$ so that the last inequality can be rewritten as

$$
\begin{aligned}
h_{\Pi_{1} K}\left(u_{1}\right)+h_{\Pi_{1} K}\left(u_{2}\right)-h_{\Pi_{1} K}\left(u_{1}+u_{2}\right) \geq & \\
& \frac{4 \cos \theta}{n-1} \int_{S^{n-3}} \int_{0}^{\infty} H_{K}(r, z)\left(\frac{\cos ^{n-2} \theta}{\left[1+r^{2} \cos ^{2} \theta\right]^{\frac{n}{2}}}-\frac{1}{\left[1+r^{2}\right]^{\frac{n}{2}}}\right) r^{n-3} d r d z .
\end{aligned}
$$

As a convex function of $r$, for every $z \in S^{n-3}, \frac{\partial H_{K}(r, z)}{\partial r}$ exists almost everywhere. Since $\frac{d}{d r}\left(\frac{r^{n-2}}{(n-2)\left(1+r^{2}\right)^{\frac{n-2}{2}}}\right)=\frac{r^{n-3}}{\left(1+r^{2}\right)^{\frac{n}{2}}}$ and $\frac{d}{d r}\left(\frac{r^{n-2}}{(n-2)\left(1+r^{2} \cos ^{2} \theta\right)^{\frac{n-2}{2}}}\right)=\frac{r^{n-3}}{\left(1+r^{2} \cos ^{2} \theta\right)^{\frac{n}{2}}}$, integration by parts yields

$$
\begin{aligned}
h_{\Pi_{1} K}\left(u_{1}\right)+ & h_{\Pi_{1} K}\left(u_{2}\right)-h_{\Pi_{1} K}\left(u_{1}+u_{2}\right) \geq \\
& \frac{4 \cos \theta}{n-1} \int_{S^{n-3}}\left[H_{K}(r, z) \frac{r^{n-2}}{n-2}\left(\frac{\cos ^{n-2} \theta}{\left[1+r^{2} \cos ^{2} \theta\right]^{\frac{n-2}{2}}}-\frac{1}{\left[1+r^{2}\right]^{\frac{n-2}{2}}}\right)\right]_{0}^{\infty} d z \\
- & \frac{4 \cos \theta}{n-1} \int_{S^{n-3}}^{\infty} \int_{0}^{\infty} \frac{\partial H_{K}(r, z)}{\partial r} \frac{r^{n-2}}{n-2}\left(\frac{\cos ^{n-2} \theta}{\left[1+r^{2} \cos ^{2} \theta\right]^{\frac{n-2}{2}}}-\frac{1}{\left[1+r^{2}\right]^{\frac{n-2}{2}}}\right) d r d z .
\end{aligned}
$$


Note that $H_{K}(r, z) / \sqrt{1+r^{2}}$ is the value of the support function $h_{K}$ at a point of the unit sphere and so the first integral in the above formula vanishes, since

$$
\lim _{r \rightarrow+\infty} \frac{r^{n-2} \cos ^{n-2} \theta \sqrt{1+r^{2}}}{\left[1+r^{2} \cos ^{2} \theta\right]^{\frac{n-2}{2}}}-\frac{r^{n-2}}{\left[1+r^{2}\right]^{\frac{n-3}{2}}}=0 .
$$

In order to estimate the second integral we first note that the integrand is nonnegative. By the definition of $H_{K}, \frac{\partial H_{K}(r, z)}{\partial r}$ is the derivative of $h_{K}$ at the point $x=\left(0,1, y_{2}(r, z), \ldots, y_{n-1}(r, z)\right)$ in the direction $z$ and then (see [11], Th. 1.7.2) it is the value of the support function at $z$ of the support set of $K$ with exterior normal vector $x /\|x\|$. Hence, by considering the projection of $K$ onto the two dimensional plane spanned by $z$ and $v=\frac{u_{1}-u_{2}}{\left\|u_{1}-u_{2}\right\|}=(0,1,0, \ldots, 0)$, we obtain

$$
\frac{\partial H_{K}(r, z)}{\partial r} \geq h_{K}(z)-\frac{h_{K}(v)}{r}
$$

for all $r \geq r_{0}(z)=\frac{h_{K}(v)}{h_{K}(z)}$, where, for simplicity, by identifying $S^{n-3}$ with $S^{n-1} \cap u_{1}^{\perp} \cap u_{2}^{\perp}$, we wrote $z$ instead of $\left(0,0, y_{2}(1, z), \ldots, y_{n-1}(1, z)\right)$.

By (9) we obtain

$$
\begin{aligned}
& h_{\Pi_{1} K}\left(u_{1}\right)+h_{\Pi_{1} K}\left(u_{2}\right)-h_{\Pi_{1} K}\left(u_{1}+u_{2}\right) \geq \\
& \frac{4 \cos \theta}{(n-1)(n-2)} \int_{S^{n-3}} H_{K}(1, z) \int_{r_{0}(z)}^{\infty}\left(1-\frac{r_{0}(z)}{r}\right)\left(\left(\frac{r}{\sqrt{1+r^{2}}}\right)^{n-2}-\left(\frac{r \cos \theta}{\sqrt{1+r^{2} \cos ^{2} \theta}}\right)^{n-2}\right) d r d z .
\end{aligned}
$$

It is not difficult to verify that

$$
\begin{aligned}
& \int \frac{r^{n-3}}{\left(1+r^{2}\right)^{\frac{n-2}{2}}} d r=\left\{\begin{array}{cc}
\ln \left(r+\sqrt{1+r^{2}}\right)-\sum_{k=0}^{\frac{n-5}{2}} \frac{r^{2 k+1}}{(2 k+1)\left(1+r^{2}\right)^{\frac{2 k+1}{2}}} & \text { for n odd } \\
\ln \left(\sqrt{1+r^{2}}\right)-\sum_{k=1}^{\frac{n-4}{2}} \frac{r^{2 k}}{2 k\left(1+r^{2}\right)^{k}} & \text { for } \mathrm{n} \text { even }
\end{array}\right. \\
& \int \frac{r^{n-2}}{\left(1+r^{2}\right)^{\frac{n-2}{2}}} d r=\left\{\begin{array}{cc}
\frac{(n-3) ! !}{(n-4) ! !} \sqrt{1+r^{2}}-\frac{1}{n-1} \sum_{k=1}^{\frac{n-3}{2}} \frac{(n-1)(n-3) \ldots(2 k+2) r^{2 k}}{(n-4)(n-6) \ldots(2 k-1)\left(1+r^{2}\right)^{\frac{2 k-1}{2}}} & \text { for } \mathrm{n} \text { odd } \\
\frac{(n-3) ! !}{(n-4) ! !}(r-\arctan r)-\frac{1}{n-1} \sum_{k=1}^{\frac{n-4}{2}} \frac{(n-1)(n-3) \ldots(2 k+3) r^{2 k+1}}{(n-4)(n-6) \ldots 2 k\left(1+r^{2}\right)^{k}} & \text { for } \mathrm{n} \text { even . }
\end{array}\right.
\end{aligned}
$$

These formulas allow to rewrite the above inequality as follows:

$$
\begin{aligned}
h_{\Pi_{1} K}\left(u_{1}\right)+ & h_{\Pi_{1} K}\left(u_{2}\right)-h_{\Pi_{1} K}\left(u_{1}+u_{2}\right) \geq \\
& \frac{4 \cos \theta}{(n-1)(n-2)} \int_{S^{n-3}} H_{K}(1, z) \quad\left[F\left(\frac{1}{\cos \theta}\right)-F(1)\right] d z,
\end{aligned}
$$


where

$F(x)=\frac{(n-3) ! !}{(n-4) ! !} \sqrt{x^{2}+r_{0}(z)^{2}}-r_{0}(z) \ln \left(r_{0}(z)+\sqrt{x^{2}+r_{0}(z)^{2}}\right)+\sum_{k=1}^{\frac{n-5}{2}} \frac{\left(1-\lambda_{k}\right) r_{0}(z)^{2 k}}{(2 k-1)\left(x^{2}+r_{0}(z)^{2}\right)^{\frac{2 k-1}{2}}}$,

for $n$ odd, with $\lambda_{k}=\frac{(n-3)(n-5) \ldots(2 k+2)}{(n-4)(n-6) \ldots(2 k+1)}$ and

$$
F(x)=\frac{(n-3) ! !}{(n-4) ! !}\left[\frac{\pi}{2} x-x \arctan \frac{r_{0}(z)}{x}\right]-\frac{r_{0}(z)}{2} \ln \left(x^{2}+r_{0}(z)^{2}\right)+\sum_{k=1}^{\frac{n-6}{2}} \frac{\left(1-\mu_{k}\right) r_{0}(z)^{2 k+1}}{2 k\left(x^{2}+r_{0}(z)^{2}\right)^{k}}
$$

for $n$ even, with $\mu_{k}=\frac{(n-3)(n-5) \ldots(2 k+3)}{(n-4)(n-6) \ldots(2 k+2)}$.

Note that $\lambda_{k}$ and $\mu_{k}$ are not smaller than 1 , for every $k$.

If $n$ is odd, then simple computations give

$$
F^{\prime}(x) \geq \frac{x}{\sqrt{x^{2}+r_{0}(z)^{2}}}\left[\frac{(n-3) ! !}{(n-4) ! !}-\frac{r_{0}(z)}{r_{0}(z)+\sqrt{x^{2}+r_{0}(z)^{2}}}\right] .
$$

Since the right-hand side above is an increasing function with respect to $x$, by the mean value theorem, we have that

$$
\begin{aligned}
& h_{\Pi_{1} K}\left(u_{1}\right)+h_{\Pi_{1} K}\left(u_{2}\right)-h_{\Pi_{1} K}\left(u_{1}+u_{2}\right) \geq \\
& \quad \frac{4(n-3) ! !}{(n-1)(n-2) ! !}(1-\cos \theta) \int_{S^{n-3}} \frac{h_{K}(z)^{2}}{h_{K}(v)+\sqrt{h_{K}(v)^{2}+h_{K}(z)^{2}}} d z,
\end{aligned}
$$

which is nothing but inequality (6).

If $n$ is even, then, for $x \geq 1$,

$$
F^{\prime}(x) \geq \frac{(n-3) ! !}{(n-4) ! !}\left(\frac{\pi}{2}-\arctan r_{0}(z)\right) \geq \frac{(n-3) ! !}{(n-4) ! !} \frac{1}{r_{0}(z)+\sqrt{1+r_{0}(z)^{2}}},
$$

where we used inequalities $\arctan \frac{1}{x} \geq \frac{1}{\sqrt{1+x^{2}}} \geq \frac{1}{x+\sqrt{1+x^{2}}}$. Hence, (6) holds in the even case, too.

Corollary 2.2. If $\operatorname{dim} K>2$, then the body $\Pi_{1} K$ has no singular points.

We recall that a singular point of a convex body is a boundary point where the normal cone has dimension greater than one (see [11], p. 73).

Proof. Assume that $\Pi_{1} K$ has a singular point $p$ and let $u_{1}, u_{2}$ be two distinct directions belonging to the normal cone of $\Pi_{1} K$ at $p$. This implies that the left-hand side in (6) vanishes and consequently the same happens for $I\left(K ; u_{1}, u_{2}\right)$. By the continuity of $h_{K}$ we deduce that such a function must vanish everywhere in $u_{1}^{\perp} \cap u_{2}^{\perp}$.

Hence, $K$ lies in the plane spanned by $u_{1}$ and $u_{2}$. 
Recall that the projection body of order one of a segment is an $(n-1)$-dimensional ball centered at the origin.

If $K$ is two dimensional, then $D K$ is a zonoid and $\Pi_{1} K$ is a sum (or a limit of sums) of $(n-1)$-dimensional balls. Furthermore, the section of $\Pi_{1} K$ with the two dimensional plane containing $D K$ is similar to $D K$.

In particular, by Corollary 2.2 and the above remarks about special cases, we conclude that $\Pi_{1} K$ has no point with an $n$-dimensional normal cone, shortly, has no vertex. As Rolf Schneider pointed out to the authors, the same conclusion can be obtained by the following alternative argument. If $\Pi_{1} K$ has a vertex, then the first order area measure of $K$ vanishes on a neighborhood of some equator (see [10], Lemma 6.1). This contradicts the result proved by Fedotov [4], stating that the support of the first order area measure of an $n$-dimensional convex body is arcwise connected if $n>2$ (see also [11], Note 7.1.6).

Fix a point $p$ on the boundary of $\Pi_{1} K$ and denote by $n(p)$ the outward unit normal vector to $\partial \Pi_{1} K$ at $p$. For any $v$ orthogonal to $n(p)$, we denote by $\alpha(p ; v)$ the two dimensional plane through $p$ spanned by $n(p)$ and $v$. We define $r(p ; v)$ as the radius of curvature of the projection of $\Pi_{1} K$ onto $\alpha(p ; v)$ at $p$, if it exists.

Corollary 2.3. If $\operatorname{dim} K>2$, then

$$
r(p ; v) \geq \frac{2(n-3) ! !}{(n-1)(n-2) ! !} \int_{S^{n-1} \cap n(p) \perp \cap v^{\perp}} \frac{h_{K}(z)^{2}}{h_{K}(n(p))+\sqrt{h_{K}(n(p))^{2}+h_{K}(z)^{2}}} d z .
$$

Proof. The statement is a straightforward consequence of (6), by taking into account that

$$
r(p ; v)=\lim _{\varepsilon \rightarrow 0} \frac{h_{\Pi_{1} K}(n(p)+\varepsilon v)+h_{\Pi_{1} K}(n(p)-\varepsilon v)-2 h_{\Pi_{1} K}(n(p))}{\varepsilon^{2}},
$$

which can be deduced, for example, from formula (2), p. 71, in [2].

Note that if $\operatorname{dim} K>2$, then the support of the area measure $S_{1}\left(\Pi_{1} K ; \cdot\right)$ coincides with $S^{n-1}$. Indeed, if $\Pi_{1} K$ is $C_{+}^{2}$, then, for every open subset $\omega$ of $S^{n-1}, S_{1}\left(\Pi_{1} K ; \omega\right)$ is the integral over $\omega$ of the arithmetic mean of the principal radii of curvature (see formula (4.2.20) in [11]). Hence, $S_{1}\left(\Pi_{1} K ; \omega\right)=0$ implies that every radius of curvature at every point with normal in $\omega$ is zero. This contradicts Corollary 2.3. If $\Pi_{1} K$ is not $C_{+}^{2}$, then an approximation argument based on Weil's result in [13] (see also [11], p. 119) leads to the same conclusion.

\section{An EXISTENCE PROBLEM}

In this section we deal with a version of Problem 1.1 when the data are available only for finitely many hyperplanes. The aim is to show that some existence results obtained in [3] for the $(n-1)$ th area measure are not reproducible for the one of the first order.

In particular, the results contained in [3] imply that, for arbitrary fixed directions $u_{1}$, $u_{2}, \ldots, u_{s}, v \in S^{2}$, with $u_{j} \in v^{\perp}, j=1,2, \ldots, s$, and convex body $K \subset \mathbb{R}^{3}$, there exists 
a convex origin-symmetric prism $P$, such that each facet is parallel to two directions from $u_{1}, u_{2}, \ldots u_{s}, v$, and

$$
\begin{aligned}
V_{2}\left(P \mid v^{\perp}\right) & =V_{2}\left(K \mid v^{\perp}\right), \\
V_{2}\left(P \mid u_{j}^{\perp}\right) & =V_{2}\left(K \mid u_{j}^{\perp}\right), \quad \text { for } j=1,2, \ldots, s .
\end{aligned}
$$

By a limit process we obtain that, for every convex body $K$ and direction $v$, there exists a convex origin-symmetric cylinder $C$, with axis parallel to $v$, such that

$$
\begin{aligned}
V_{2}\left(C \mid v^{\perp}\right) & =V_{2}\left(K \mid v^{\perp}\right), \\
V_{2}\left(C \mid u^{\perp}\right) & =V_{2}\left(K \mid u^{\perp}\right), \quad \text { for every } u \in v^{\perp} .
\end{aligned}
$$

We now consider the case $i=1$ and show that, for $n=3$, the existence of a convex origin-symmetric cylinder $C$, whose axis is parallel to $v$, such that

$$
\begin{aligned}
V_{1}\left(C \mid v^{\perp}\right) & =V_{1}\left(K \mid v^{\perp}\right), \\
V_{1}\left(C \mid u^{\perp}\right) & =V_{1}\left(K \mid u^{\perp}\right), \quad \text { for every } u \in v^{\perp},
\end{aligned}
$$

is not guaranteed, i.e. may depend on $K$ and $v$.

Assume $C=a v+L$, where $a$ is a positive number and $L$ is a two dimensional originsymmetric convex body contained in $v^{\perp}$. Conditions (10) and (11) can be written as

$$
\frac{1}{2} \int_{S^{2} \cap v^{\perp}} h_{L}(u) d u=h_{\Pi_{1} K}(v)
$$

and

$$
a+2 h_{L}(u)=h_{\Pi_{1} K}(u \times v),
$$

for every $u \in S^{2} \cap v^{\perp}$, where $\times$ stands for the standard cross product. By integrating (13) with respect to $u$ and using (12) we obtain

$$
a=\frac{1}{2 \pi} \int_{S^{2} \cap v^{\perp}} h_{\Pi_{1} K}(u) d u-\frac{2}{\pi} h_{\Pi_{1} K}(v)
$$

and

$$
h_{L}(u)=\frac{1}{2} h_{\Pi_{1} K}(u \times v)-\frac{1}{4 \pi} \int_{S^{2} \cap v^{\perp}} h_{\Pi_{1} K}(z) d z+\frac{1}{\pi} h_{\Pi_{1} K}(v) .
$$

Note that for every convex body $K$, the right-hand side in (14) is nonnegative. Indeed, $K \mid u^{\perp}$ is inscribed in a rectangle with edge lengths $2 h_{K}(v)$ and $2 h_{K}(u \times v)$ and so its perimeter is not less than twice the length of one of the edges. Therefore,

$$
\int_{S^{2} \cap v^{\perp}} h_{\Pi_{1} K}(u) d u \geq 2 \int_{S^{2} \cap v^{\perp}} h_{K}(u \times v) d u=4 h_{\Pi_{1} K}(v) .
$$


On the other hand, the perimeter of $K \mid u^{\perp}$ does not exceed that of the rectangle. Consequently, the right-hand side in (15) is nonnegative too. Indeed,

$$
\int_{S^{2} \cap v^{\perp}} h_{\Pi_{1} K}(u) d u \leq 2 \int_{S^{2} \cap v^{\perp}} h_{K}(u \times v) d u+4 \pi h_{K}(v) \leq 4 h_{\Pi_{1} K}(v)+2 \pi h_{\Pi_{1} K}(u \times v) .
$$

Nevertheless, it turns out that there are convex bodies $K$ for which the function $h_{L}$ in (15), extended everywhere in $\mathbb{R}^{n-1}$ as a positively homogeneous function, is not a support function. To see this, we take the segment $K$ whose endpoints are $-w$ and $w$, where $w=(0, \cos \varphi, \sin \varphi)$, and show that $h_{L}$ is not convex for suitable $\varphi \in[0, \pi / 2]$. Indeed the quantity

$$
h_{L}(1 / \sqrt{2}, 1 / \sqrt{2}, 0)+h_{L}(1 / \sqrt{2},-1 / \sqrt{2}, 0)-2 h_{L}(1 / \sqrt{2}, 0,0)
$$

assumes negative values for $\varphi$ close to 0 . Since $h_{\Pi_{1} K}(x)=2\|x \times w\|$, by (15) the quantity (16) can be explicitly written as

$$
F(\varphi)=\sqrt{2}\left[\sqrt{2-\cos ^{2} \varphi}-1-\frac{\sqrt{2}-1}{2 \pi}\left(\int_{0}^{2 \pi} \sqrt{1-\cos ^{2} \varphi \sin ^{2} \theta} d \theta-4 \cos \varphi\right)\right]
$$

and it is easy to verify that $F(0)=0$ and $F^{\prime}(\varphi)$ is negative for sufficiently small positive values of $\varphi$.

The freedom in the choice of $\varphi$ provides many different segments such that $F$ is negative and so the corresponding function $h_{L}$ is not convex. The linearity of $\Pi_{1}$ with respect to Minkowski addition implies that sums of such segments give rise to a function $h_{L}$ such that (16) is negative. In other words, one can construct zonotopes for which no cylinder satisfying (10) and (11) exists.

This conclusion implies also that there are directions $u_{1}, u_{2}, \ldots u_{s}, v$, with $u_{j} \in v^{\perp}, j=$ $1,2, \ldots, s$, and convex bodies $K$ such that no convex origin-symmetric prism $P$ exists, with each facet parallel to two of the chosen directions, satisfying

$$
V_{1}\left(P \mid u^{\perp}\right)=V_{1}\left(K \mid u^{\perp}\right) \text {, for every } u \in\left\{u_{1}, u_{2}, \ldots, u_{s}, v\right\} \text {. }
$$

As a final remark, we want to emphasize that the convexity of $h_{L}$ in (15) is equivalent to the following inequality:

$$
\frac{h_{\Pi_{1} K}\left(u_{1}\right)+h_{\Pi_{1} K}\left(u_{2}\right)-h_{\Pi_{1} K}\left(u_{1}+u_{2}\right)}{2-\left\|u_{1}+u_{2}\right\|} \geq \frac{1}{2 \pi} \int_{S^{2} \cap v^{\perp}} h_{\Pi_{1} K}(z) d z-\frac{2}{\pi} h_{\Pi_{1} K}(v),
$$

for every $u_{1}, u_{2} \in S^{2} \cap v^{\perp}, u_{1} \neq u_{2}$. Inequality (17) is of the same kind as (6), which turns out to hold for every convex body.

\section{REFERENCES}

[1] Ch. Berg, Corps convexes et potentiels sphériques, Danske Vid. Selskab. Mat.-fys. Medd. 37 (1969), 6.

[2] T. Bonnesen and W. Fenchel, Theory of Convex Bodies, BCS Associates, Moscow, ID, 1987.

[3] S. Campi, A. Colesanti and P. Gronchi, Convex bodies with extremal volumes having prescribed brightness in finitely many directions, Geom. Dedicata 57 (1995), 121-133. 
[4] V. P. Fedotov, Polar representation of a convex compactum (in Russian), Ukrain. Geom. Sb. 25 (1982), $137-138$.

[5] W. J. Firey, Christoffel's problem for general convex bodies, Mathematika 15 (1968), 7-21.

[6] R. J. Gardner, Geometric Tomography, Cambridge University Press, New York, 2nd ed., 2006.

[7] R. J. Gardner and P. Milanfar, Reconstruction of convex bodies from brightness functions, Discrete Comput. Geom. 29 (2003), 279-303.

[8] H. Groemer, Geometric Applications of Fourier Series and Spherical Harmonics, Cambridge University Press, New York, 1996.

[9] P. Guan and X. N. Ma, The Christoffel-Minkowski problem I: Convexity of solutions of a Hessian equation, Invent. Math. 151 (2003), 553-577.

[10] R. Schneider, Über eine Integralgleichung in der Theorie der konvexen Körper, Math. Nachr. 44 (1970), 55-75.

[11] R. Schneider, Convex bodies: The Brunn-Minkowski theory, Cambridge University Press, Cambridge, 1993.

[12] R. Schneider and W. Weil, Zonoids and related topics, in Convexity and its Applications (eds P. M. Gruber and G. M. Wills), Birkhäuser, Basel (1983), 296-317.

[13] W. Weil, Ein Aproximationssatz für konvexe Körper, Manuscripta Math. 8 (1973), 335-362.

Dipartimento di Ingegneria dell'Informazione, Università degli Studi di Siena, Via Roma 56, 53100 SiEnA, ITALY

E-mail address: campi@dii.unisi.it

Dipartimento di Matematica e Applicazioni Per l'Architettura, Università degli Studi di Firenze, Piazza Ghiberti 27, 50122 Firenze, Italy

E-mail address: paolo@fi.iac.cnr.it 